\title{
Zinc removal from wastewater using hydrogel modified biochar
}

\begin{abstract}
In this study, hydrogel-(rice husk) biochar composite (HBC-RH) was prepared using acrylamide (AAm) as monomer, with N.Nômethylenebisacrylamide (MBA) as crosslinker, ammonium persulfate (APS) as initiator and rice husk biochar (RHB). The synthesized hydrogel-(rice husk) biochar composite was characterized for swelling capacity andthen utilized for the removal of zinc from wastewater. The optimum batch experimental conditions for this study were determined by evaluating the effect of solution $\mathrm{pH}$, adsorbent dosage, adsorbate initial concentration and contact time. Langmuir isotherm best fitted the HBC-RH sorption of Zinc whereas the kinetic data were best described by pseudo second-order. The maximum monolayer sorption capacity of $\mathrm{HBC}-\mathrm{RH}$ for zinc was $35.75 \mathrm{mg} / \mathrm{g}$.
\end{abstract}

Keyword: Adsorption; Biochar; Hydrogel; Isotherm; Kinetics; Zinc 